\title{
Des loups et des hommes : vers une rébellion de la nature
}

Analyse de la nouvelle Le Loup-garou (1926) d'August Gailit

Wolves and men: towards a rebellion of nature

Hundid ja inimesed: looduse mässust

\section{Aude Courty}

\section{OpenEdition}

\section{Journals}

Édition électronique

URL : https://journals.openedition.org/efo/3663

DOI : $10.4000 /$ efo.3663

ISSN : 2275-1947

Éditeur

INALCO

\section{Édition imprimée}

Date de publication : 1 janvier 2014

ISBN : 978-2-343-05394-3

ISSN : 0071-2051

\section{Référence électronique}

Aude Courty, «Des loups et des hommes : vers une rébellion de la nature ", Études finno-ougriennes [En ligne], 46 | 2014, mis en ligne le 13 octobre 2015, consulté le 08 juillet 2021. URL : http:// journals.openedition.org/efo/3663; DOI : https://doi.org/10.4000/efo.3663

Ce document a été généré automatiquement le 8 juillet 2021.

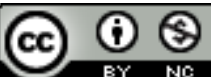

Études finno-ougriennes est mis à disposition selon les termes de la Licence Creative Commons Attribution - Pas d'Utilisation Commerciale 4.0 International. 


\title{
Des loups et des hommes : vers une rébellion de la nature
}

\author{
Analyse de la nouvelle Le Loup-garou (1926) d'August Gailit \\ Wolves and men: towards a rebellion of nature \\ Hundid ja inimesed: looduse mässust
}

Aude Courty

1 Si August Gailit (1891-1960) a débuté sa carrière d'écrivain estonien par des nouvelles et des romans de facture néo-romantique, il n'a jamais vraiment renoncé à ses thèmes de prédilection, l'étrange et l'insolite. Le Loup-garou ne déroge pas à la règle, campant un héros en proie au mal-être et à la misanthropie et pour lequel la lycanthropie fantastique se révèle salutaire. Le narrateur, Enrik, fils d'un habitant du marais d'Urgvee, ne partage avec son père qu'une haine réciproque. Nostalgique des régions enchanteresses situées par-delà les frontières du marécage, Enrik trouve cependant un second souffle dans son amour pour Hundva, fille de la vieille Ulla, sorcière et amie du père d'Enrik. Lorsqu'Enrik est accusé, à la place de Hundva, de lycanthropie, cette dernière essaie de le persuader de laisser s'exprimer sa part animale. Face au refus d'Enrik, elle se tourne alors vers le père, qu'elle épouse devant pléthore de villageois. Pour éveiller à nouveau l'intérêt de Hundva, Enrik consent donc à se changer en loup sauvage, à la faveur d'une métamorphose allant crescendo. Dans quelle mesure la lycanthropie incarne-t-elle une nature en révolte contre l'impérialisme des hommes? Dans un premier temps, le motif du loup-garou se révèle sous différents aspects tels que la figure du meneur de loups, la dimension sociale de la lycanthropie et ses possibles bienfaits. Dans un deuxième temps, sont mis en avant les deux mouvements complémentaires du texte : une animalisation de l'homme versus une humanisation de la nature. Si l'homme ressemble à s'y méprendre à ses animaux, tour à tour réifié, dépossédé de son être et désindividualisé, sa transformation témoigne d'une volonté de communier avec la nature. Dans un troisième temps, le changement du personnage en loup se veut la punition infligée pour les exactions commises envers mère-nature. Pernicieux prédateur, l'homme est vu en tant que créature monstrueuse, un zombie condamné au non-être, vain et sans but. 
2 Le loup-garou, en plus de donner son nom à l'œuvre d'August Gailit, apparaît sous diverses formes au cours de la nouvelle à la faveur de certaines références littéraires disséminées entre les lignes du texte. Tout d'abord, le lycanthrope est associé à un décor désolé, inanimé, apte à répondre à la mélancolie, au spleen du narrateur Enrik. Les nuages évoquent de la sorte le couvercle baudelairien (Baudelaire 1972, p. 92) écrasant le personnage, immergé dans ses angoisses existentielles, sa solitude et son désir d'ailleurs: "Au-dessus de ma tête se ferment des nuages de plomb, aucun oiseau ne chante ni ne gazouille sous ce plafond. $»^{1}$ Cette citation n'est pas sans rappeler le premier couplet de la ballade «La Belle Dame sans merci » (1884) de John Keats, un peu moins d'un siècle avant la parution de la nouvelle de Gailit :

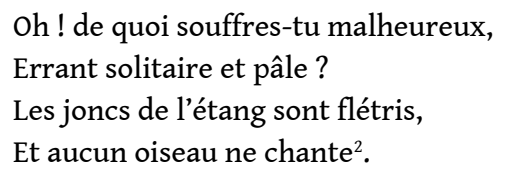

3 Chez Keats, le personnage masculin - tout comme le héros de Gailit - se perd dans les filets de la femme aimée, affiliée à une nature ravisseuse et close telle un guet-apens.

Ensuite, le loup-garou, au travers du personnage de Hundva, se confond avec la figure du meneur de loups. Le meneur de loups, qui peut lui-même être un ancien loup-garou, le chef d'une meute de lycanthropes ou un sorcier, est un ermite vivant retiré dans les bois et s'adressant aux fauves à l'aide de formules magiques ou dans une langue inusitée. En outre, selon plusieurs légendes, la peau de loup est l'attribut fabuleux du meneur de loups. Dans Hugues le Loup (1866) d'Erckmann-Chatrian, le comte de Nideck, après avoir endossé une toison lupine, hurle comme une bête et se croit devenu loup, tel qu'en témoigne ce portrait :

Le comte de Nideck - que je croyais mourant -, revêtu d'une énorme peau de loup, dont la mâchoire supérieure s'avançait en visière sur son front, les griffes sur ses épaules, et dont la queue traînait derrière lui sur les dalles (Erkmann-Chatrian, Contes III, p. 199).

5 L'accessoire vestimentaire agit donc en tant que déclencheur nécessaire de la métamorphose ; de même que dans la nouvelle de Gailit :

Puis, quand j'ai fini de manger, elle [Hundva] apporte une peau de loup et la jette sur mes épaules. Elle psalmodie longuement d'étranges paroles et dessine des signes incompréhensibles. Puis elle lance un cri, sonore et impérieux : "Cours, fils de loup!"

6 Ici, nulle pleine lune présidant à la transformation, ni fleurs maléfiques instigatrices de métamorphose, comme chez Robert Bloch et ses monstres lunatiques (Bloch 1998). En outre et contrairement à certains récits contemporains de loups-garous, tel que le roman-calendrier Cycle of the Werewolf (1983) de Stephen King, le lycanthrope ne conserve pas de caractères humains - si ce n'est son mode de pensée - qui participent de son hybridité. Bien que le changement se veuille progressif (Enrik fait de fréquentes allusions à ses dents qui ne cessent de pousser), il s'agit de se transformer irréversiblement en loup. Enrik choisit en effet de ne pas redevenir homme, à l'inverse de Hundva qui vogue inlassablement entre les deux états. De ce fait, il perd toute notion de langage humain :

"Haouuu !" hurlé-je, et le marais me répond d'un écho plein d'entrain.

7 Le pendant littéraire slave dénonce une défaillance d'expression similaire dans le roman Le Livre sacré du loup-garou (2004) de Viktor Pelevine 3 . Une fois métamorphosé, le 
loup-garou Alexandre Seryj doit alors renoncer au don de la parole auquel il substitue une nouvelle forme de langage :

Les capacités de communication qu'il lui restait n'étaient pas suffisantes pour exprimer des mouvements compliqués de l'âme, mais il pouvait répondre par l'affirmative ou la négative. Pour "oui", il lançait un bref grognement sourd : "Rrr !" Pour "non", il émettait un son à mi-chemin entre un hurlement et un bâillement : “Wou-u-u !" (Pelevine 2009, p. 190).

8 Par ailleurs, dans le texte de Gailit, la lycanthropie s'organise telle une micro-société. Effectivement, elle est vue sous un jour communautaire, d'abord au sein de la famille (Enrik et son père, Ulla et sa fille), puis prenant les dimensions du village entier :

Vieilles et vieillards sont venus, antiques habitants d'Urgvee semblables à des spectres, des visions [...]. Ils ont la pipe au bec et ils mangent, ils fument et boivent à la fois, avec une avidité et une hâte animales.

Le village évoque ainsi la meute, dominée par l'instinct grégaire :

Je ne suis pas seul, marais et tourbières sont pleins de jeunes loups. Parfois nous nous regroupons et courons en meutes.

10 La lycanthropie apparaît alors moins comme une malédiction, que comme une bénédiction, un remède à la solitude et un synonyme de liberté. S'opère de fait un véritable retournement de situation; consécutivement à sa métamorphose, le héros de Gailit confie :

Il me semble être délivré du sortilège, je me sens soudain libre et joyeux.

$\mathrm{Au}$ contraire, l'humanité s'avère être un handicap rebutant, une condition humiliante :

Je ne veux tout simplement plus être un homme.

12

Et c'est ici que la frontière entre lycanthrope et humain se fait de plus en plus ténue ; le vrai loup-garou n'est pas la bête, mais l'homme violent, prédateur, assassin, dont l'archétype reste le père du narrateur, qui bat jusqu'au sang sa jeune épousée et la garde recluse. L'œuvre de Gailit se veut donc hantée par un double mouvement, pareil à une inversion des rôles : l'abêtissement de l'homme et l'humanisation du fauve, de la nature.

Régulièrement dans le texte, les personnages humains souffrent d'une comparaison avec leurs voisins animaux : les habitants du marais sont par exemple comparés à des serpents venimeux, les femmes à des oiseaux gouailleurs (pies, chouettes effraies), le père est comparé à un chien et la métaphore du loup revient souvent au fil de la nouvelle. Plus spécifiquement, l'image du serpent est récurrente comme étant l'emblème de l'animal séducteur, tentateur, se livrant à la séduction de la sauvagerie, de l'instinct bestial, soulignant la tentation de la métamorphose. La femme, particulièrement, en la personne d'Hundva, se veut cette créature perfide, lascive et perverse, la louve sanguinaire qui perd et corrompt les hommes (les villageois, le père, Enrik) et exhorte à un amour fauve :

"Tu vas peut-être te mettre à me mordre ?" demande-t-elle.

14 Elle réveille leurs inclinations primitives et primordiales, et les fait se nourrir tels des outre-mangeurs :

ils rongent les quartiers de viande fumante, comme des charognards.

15 De plus, l'animalité prend la forme d'une injonction, d'un ordre. L'homme ne peut transiger et doit composer, tôt ou tard, avec sa part bestiale :

sois un animal, comme nous tous ici. 
D’où le déni, le dégoût de l'humanité exprimé par le personnage central :

Je conserve en moi de la pitié et de l'amour, des sentiments humains, répugnants.

u-delà de la comparaison animalière, il y a également un parallélisme d'apparence entre l'homme et son bétail. Aussi, tout comme le personnage du père est " pauvre et osseux », son cheval est-il " osseux » et ses agneaux " faméliques ». Plus généralement, l'être humain est réifié. En exemple, le narrateur se compare fréquemment à un arbre :

J'ai grandi comme un tremble, dont les feuilles argentées frémissent sans cesse.

Il en fait de même auprès du personnage de Kristjuhan, affublé d'un corps végétal pleurant des larmes de sève. De la sorte, il témoigne de la perte de son humanité d'une part et de son enracinement à un destin inéluctable, d'autre part. Digne d'un héros romantique, Enrik apparaît ainsi comme un élément du décor, englué dans sa condition et dépourvu d'animation, de vitalité. L'être humain se désolidarise, se dissout pour renaître sous l'enveloppe d'un corps élémentaire, fait de terre, d'eau et d'air, et non plus de chair et de sang :

J'ai grandi dans le marais d'Urgvee comme un pin rabougri, les racines dans la boue Je suis comme un poisson jeté dans la glace, les nageoires cassées et les branchies pleines de morceaux de glace ou encore

[L'homme] prend la fuite en nous voyant comme un oiseau prend son envol.

L'humain est par la suite désindividualisé et dépossédé de son être propre (« Tout est à eux, même moi avec ma peur »), dont l'identité singulière s'efface au profit de celle, collective, de l'espèce ; d'où la comparaison des hommes à des fourmis, soldats sacrifiés au nom de la survie du groupe. Sur une autre échelle, si le loup incarne un certain idéal de liberté, il n'en reste pas moins également l'élément d'une meute régie par l'instinct grégaire.

21 En retour, si l'homme est abêti, la nature - et ses différents acteurs - sont semblablement humanisés, suivant le concept allemand de Naturphilosophie (fin du $\mathrm{XVIII}^{\mathrm{e}}$ siècle) qui encourageait l'homme à reconnaître son image dans la nature et à trouver en lui-même un paysage, selon l'osmose du moi avec l'environnement naturel. Ainsi, le vent, les brumes et le marécage murmurent, sifflent et se gaussent à l'approche du narrateur, le fauve est «assassin[é]» comme un homme d'après Enrik, « crach[e] comme un humain " d'après le personnage du père. La métamorphose peut alors traduire la volonté de se fondre en la nature, de communier avec elle :

Les animaux ont les forêts, les tourbières qui les abritent, moi je n'ai plus d'endroit où fuir votre turpitude [...]. Transforme-moi en loup, que les forêts s'ouvrent devant moi et que seule la haine des humains niche dans mon âme.

En conséquence, la lycanthropie symbolise les revendications fantastiques d'une nature en révolte contre l'impérialisme de l'homme; l'atteste ce piège naturel prêt à se refermer sur une éventuelle proie humaine :

La lune brille, la neige étincelle sous mes pas, les arbres projettent de longues ombres en tous sens, comme un épais filet qu'ils tisseraient entre eux.

La nouvelle d'August Gailit se veut enfin une lettre de dénonciation des méfaits produits par le genre humain à l'égard de la nature :

« Les hommes sont les plus vicieux des prédateurs »,

affirme Enrik. La thématique de la prédation se fait ainsi récurrente au fil du texte, tout d'abord dans le discours du narrateur lycanthrope : 
Quand elles me voient, les corneilles s'envolent comme des flèches, et les écureuils bondissent avec effroi de branche en branche [...]. Quiconque croise mon chemin est un étranger et un ennemi («l'étranger »), la peur de soi (la bête humaine) ou la peur d'un autre possible, comme
le souligne cette vision médiévale de l'univers exprimée par Hundva:

Les marais d'Urgvee s'étendent jusqu'au bout du monde, et ensuite il y a l'obscurité!

L'humain, dans toute son ambivalence, est vu comme un monstre : un loup-garou, un vampire, un zombie fantastiquement parlant, un meurtrier rationnellement parlant. Vampirique, en effet, car usant de cette attraction paranormale qu'est l'hypnose éprouvée par le narrateur, sous l'emprise de la fascinante Hundva qui le détourne de ses convictions paradisiaques :

Moi aussi je me tais ; je me tais et j'écoute le chant régulier de Hundva. J'écoute - et je commence moi aussi à douter de l'existence des pays qui se trouvent de l'autre côté des forêts et des tourbières. Peut-être les ai-je vus en rêve [...].

Vampirique, également, car reposant d'un sommeil inaltérable, tel enterré dans un cercueil à l'image du père :

Il ne veut que rester couché, bras en croix sur sa poitrine.

Vampirique, enfin, car fuyant la clarté solaire, véritable maladie des résidants du marécage :

Et un habitant d'Urgvee n'a pas le droit de partir du marais, il ne peut supporter la lumière du soleil. Là-bas il ne peut que mourir comme un troll, du fait de la fragrance des fleurs, du vent du soir, des rayons du soleil.

Ces multiples analogies justifient le motif du mort-vivant, du zombie dénué de volonté propre, qui revient par intermittence entre les lignes du texte. En exemple, le personnage du père apparaitt tel un cadavre ambulant :

Il semble bien éteint, ses étroites lèvres sont bleuies, ses cheveux blanchâtres sont rares et comme mangés par les mites.

31 Il en est de même pour Hundva, réduite à une description funeste, à un portrait mortifère, retenue en otage sous les traits d'une jumelle fantomatique, Doppelgänger ${ }^{4}$ de mauvais augure :

Elle est comme une enfant chétive, apeurée et sans vie. Parfois ses yeux sont blancs comme neige.

Plus globalement, les villageois d'Urgvee sont dépourvus de vitalité, « semblables à des spectres, des visions ", ectoplasmes incarnés dont l'épiderme se ride, les cheveux pâlissent et les doigts se font serres écorchées vives.

33 L'homme est autrement monstrueux, en sa qualité de criminel. Ainsi, le père du narrateur représente-t-il un personnage assassin et infanticide :

Les habitants d'Urgvee disent qu'il a eu une femme et une ribambelle d'enfants, mais il les a tous précipités dans le marais. Ils disent qu'il les a enterrés dans la gorge du marais, là où l'on entend un murmure liquide, sous les touffes de mousse. 
34 lycanthropique. Suivant le conte Hugues le Loup d'Erckmann-Chatrian, la lycanthropie relève d'un héritage héréditaire, d'une maladie du père, affection comportementale ou folie monomaniaque. La pathologie paternelle dériverait d'un excédent fauve du père lycanthrope. Effectivement, Sigmund Freud étudiait, sous l'angle psychanalytique, l'angoisse de son patient Serge Pankejeff, qui puisait sa source dans la scène originaire $\mathrm{du}$ coït conjugal et changeait la figure paternelle en monstre lupin ${ }^{5}$. Dans l'inconscient de l'individu et en tant qu'actant principal de la sexualité parentale, le père se faisait en effet loup et, par extension, lycanthrope. Chez Gailit, l'instance paternelle domine le fils et devient le catalyseur de la métamorphose annoncée d'Enrik.

A l'instar du loup élevant son engeance à la chasse, le père lycanthrope dompte et érige Enrik en misanthrope carnassier. Par la suite, l'homme, en tant que zombie livré à luimême, c'est-à-dire abandonné par le sorcier l'ayant ramené à la vie, n'est plus que la proie d'une errance sans fin - archétype du non-être -, une marionnette désarticulée, un pantin aux mains de la fatalité. Les détours d'Enrik dans les bois, alors qu'il décide de quitter définitivement la cabane paternelle, illustrent une métaphore de l'introspection. Le protagoniste se perd (en lui-même) et, victime de certains automatismes, tourne en rond pour finalement revenir à son point de départ, car il n'y a pas d'issue:

Qui m'a jeté un sort pour me laisser errer dans ce marais sans fin, que je ne trouve nulle échappatoire? Qui me tient et me promène, invisible et mauvais, et me fait toujours revenir sur mes pas?

Pur jouet du destin, il doit accepter de délaisser son humanité au profit d'une animalité celée, enfouie, et ainsi renaître loup. Cette errance d'Enrik symbolise un personnage sans arrêt confronté à lui-même, à ses pairs. Si le loup-garou se manifeste au seuil des mondes et des espaces - y compris ceux, anthropomorphiques, du corps - tel une ouverture des possibles, pour Enrik, aucun ailleurs n'est concevable :

le monde se limite au marais murmurant d'Urgvee.

37 Au même titre que la vie en Urgvee est un simulacre, une imitation, le monde des possibles, ailleurs ou autre part, ne sont que des mirages :

Il n'y a nulle part où aller, tout n'est qu'illusion, tout le reste est délire et folie.

Ce qui renvoie à cette vision étriquée du monde émise par Hundva (supra) et, dans une autre discipline, à l'Éternel retour nietzschéen ${ }^{6}$ qui invoque une répétition périodique de l'univers, une cosmologie opérant une redondance cyclique des événements :

Il n'y a pas de sortie, pas de destination, il n'y a que l'Éternel, l'Infini Marais.

L'homme égaré en forêt, dans ces bois qui n'ont pas l'intention de le laisser s'enfuir, met une nouvelle fois en lumière cette nature se révoltant contre des hommes carnassiers, prédateurs, lycanthropes.

Ces personnages fictifs, ces événements extraordinaires, alimentent la tonalité générale $\mathrm{du}$ texte, résolument sombre et pessimiste. Le genre humain use et abuse des ressources naturelles; mais lorsque la nature emploie ses propres armes pour se défendre, l'homme se retrouve impuissant et se résigne à renouer avec un état primitif, animal, celui de la bête en accord avec un environnement de chasse et de prédation. Pour Enrik, héros de cette histoire, la métamorphose est une bénédiction lui permettant d'échapper à une humanité autodestructrice et dégénérée. 


\section{BIBLIOGRAPHIE}

BAUDELAIRE Charles,1972, Les Fleurs du Mal, Paris : Le Livre de Poche.

BLOCH Robert, 1998, Flowers from the Moon and other lunacies, Sauk City Wisconsin : Arkham House.

ERCKMANN-CHATRIAN, « Hugues le Loup », in Contes III, disponible sur

http://beq.ebooksgratuits.com/vents/Erckmann_Chatrian_Contes_3.pdf (consulté le 03.05.2012).

FREUD Sigmund, 1990, Der Wolfsmann, Paris, Presses Universitaires de France, traduit de l'allemand par Janine Altounian et Pierre Cotet.

FROST Brian J., 2003, The Essential Guide to werewolf literature, Madison: University of Wisconsin Press.

GREGORY Constantine, 2003, The Vampire Watcher's Handbook, a guide for slayers, Paris, Le Pré aux Clercs, traduit de l'anglais par Sylvaine Charlet.

KING Stephen, 1985, Cycle of the Werewolf, New York : New American Library, en français La Nuit du loup-garou, Paris : J'ai lu, 2005, traduit de l'américain par François Lasquin.

LANDRY Jean-Marc, 2006, Le Loup, Paris : Delachaux et Niestlé (coll. Les sentiers du naturaliste). KALLAS Aino, 1990, «La fiancée du loup », in La fiancée du Loup, Paris : Viviane Hamy, traduit du finnois par Françoise Arditti, Hervé Carric, Sophie Jousselin et Eva Vingiano de Pina Martins sous la direction de Jean-Luc Moreau.

LYSøE Eric (dir.), 2004, Erckmann-Chatrian au carrefour du fantastique suivi de Histoires et contes fantastiques par Émile Erckmann-Chatrian, Strasbourg : Presses Universitaires de Strasbourg. PELEVINE Viktor, 2009, Le Livre sacré du loup-garou, Paris : Denoël, traduit du russe par Galia Ackerman et Pierre Lorrain.

RONVEL Aude, 2011, Le Loup-garou dans la Littérature contemporaine, Paris : Publibook.

RUAUD André-François, 2004, Le Dictionnaire féerique, Montpellier : l'Oxymore.

SUMMERs Montague, 2005, The Werewolf in literature, [s.l.], Kessinger Publishing's.

VILLENEUVE Roland, 1970, Loups-garous et vampires, Paris : J'ai Lu (coll. L'aventure mystérieuse).

WOTLING Patrick, 2001, Le Vocabulaire de Nietzsche, Paris : Ellipses.

\section{NOTES}

1. Les citations de la nouvelle de Gailit sont extraites d'une traduction inédite de Martin Carayol.

2. Traduction d'E. de Clermont-Tonnerre.

3. Viktor Olegovič Pelevin a écrit ce roman en russe sous le titre СВЯЩЕнНАЯ КнИГА ОБОРотня. Il a été traduit en français en 2009. L'auteur de cet article part de la version française, qui sera donc ici citée (NdE).

4. « Double » ou « sosie » en allemand. 
5. Sigmund FREUD, Der Wolfsmann, Paris, Presses Universitaires de France, 1990, traduit de l'allemand par Janine Altounian et Pierre Cotet, p. 56-57 et 100-101.

6. Ewige Wiederkehr en allemand.

\section{RÉSUMÉS}

Cet article analyse la nouvelle Le Loup-garou (1926) de l'auteur estonien August Gailit et s'efforce de montrer dans quelle mesure la lycanthropie peut être appréhendée comme la révolte de la nature contre l'impérialisme humain. Tout d'abord, le motif du loup-garou, omniprésent dans le texte, y apparaît sous différents aspects, comme la figure du meneur de loups (ce qui permet, en outre, de tisser quelques liens comparatistes avec des œuvres contemporaines telles qu'Hugues le Loup d'Erckmann-Chatrian ou Le Livre sacré du loup-garou de Viktor Pelevine) et la dimension sociale que revêt la vie en meute, remède à la solitude et synonyme de liberté. Ensuite, les deux mouvements complémentaires qui irriguent le texte sont mis en lumière: l'homme est progressivement animalisé, tandis que la nature et ses multiples acteurs sont humanisés. Se dessine ainsi un parallélisme d'apparence entre l'habitant d'Urgvee et son bétail, celui-ci étant successivement réifié et dépossédé de son être. Sa mutation témoigne cependant d'une volonté de communier avec la nature. Enfin, la nouvelle dénonce les exactions commises par le genre humain à l'égard de la nature. L'homme est vu comme un monstre horrifique, voué à errer sans but et sans raison. L'être humain s'égare dans ces bois qui se refusent à le laisser partir, dénué de toute volonté et de libre-arbitre.

This article is an analysis of the short story The Werewolf (1926) by Estonian writer August Gailit. It aims to show how werewolfery can be read as a natural reply to human imperialism. Firstly, the werewolf pattern, present throughout the text, appears in the shape of many things, like the wolf leader (which allows the creation of a comparative link with contemporary novels such as Hugues le Loup by Erckmann-Chatrian /The Man-Wolf in English/, or The sacred book of the Werewolf by Viktor Pelevine) and the social dimension of life in the pack, a remedy for loneliness and synonymous with freedom. Secondly, two complementary movements irrigating the text are brought out: the man turns gradually into an animal, while nature and its various elements turn into humans. And so, we discover some closeness between the Urgvee inhabitant's appearance and his cattle; indeed, he is dispossessed of his own being. However, his mutation testifies to this will to commune with nature. Lastly, the short story tells of human damage to nature. The man is seen as a horrible monster, doomed to wander without aim or reason. The human being loses himself in these woods, which do not want to let him go, deprived of both his will and free-will.

Selles artiklis analüüsitakse Eesti kirjaniku August Gailiti novelli „Libahunt“ (1926). Püütakse näidata, kuidas libahundina käimist võib lugeda loodusliku vastusena inimimperialismile. Esiteks esineb libahunt kogu novellis mitmest aspektist: liiderhundina (mis võimaldab novelli võrrelda moodsate romaanidega nagu Erckmann-Chatriani „Hunt-Hugues“ või Viktor Pelevini „Libahundi püha raamat") aga ka hundina karja sotsiaalse elu prismast, mis ravib üksildust ja tähendab vabadust. Teiseks tuuakse esile kaht olulist dünaamikat: inimene saab järk-järgult loomaks, kusjuures loodus koos selle erinevate elementidega muutub inimeseks. Nõnda arvatakse, et Urgvee elanik näeb välja nagu tema kariloomad: ta jääb iseenda olemusest ilma. Samas tõestab see muutumine soovi saada loodusega üheks. Lõpuks jutustab novell sellest, kuidas inimene 
loodust kahjustab. Inimest nähakse kui hirmsat koletist, kes on määratud rändama põhjusetult ja sihitult. Inimene eksib metsa, mis ei taha lasta teda lahti ja mis jätab ta tahtest ja vabast volist ilma.

\section{INDEX}

Index chronologique : XXe siècle

Keywords : Estonian literature, werewolves

Mots-clés : littérature estonienne, lycanthropie, lycanthropie

Thèmes : littérature comparée 